\title{
Diagnostic performance of isolated-check visual evoked potential versus retinal ganglion cell-inner plexiform layer analysis in early primary open-angle glaucoma
}

XiangWu Chen and YingXi Zhao*

\begin{abstract}
Background: The purpose of this study was to compare the diagnostic performance of isolated-check visual evoked potential (icVEP) with that of retinal ganglion cell-inner plexiform layer (GCILP) analysis using optical coherence tomography (OCT).

Methods: A total of 45 patients were enrolled: 25 patients with open-angle glaucoma and 20 healthy patients. All patients underwent a complete ophthalmological examination. Moreover, the OCT examination was used to analyze the structures of the GCIPL. The icVEP technique was used to detect the transmission function of the magnocellular pathway, which is mainly managed by the retinal ganglion cells. The quantitative and qualitative comparisons between the diagnostic power of GCIPL analysis and that of icVEP were performed. The areas under the receiver operating characteristic curves (AUC) of GCIPL analysis and icVEP were compared using the Clarke-Pearson method. The sensitivity and specificity of the two techniques were analyzed and compared using the McNemar test.

Results: With the quantitative comparison, the AUC of icVEP (AUC = 0.892) was higher than that of GCIPL analysis (AUC $=0.814$ ). However, there was no statistical significance between the AUCs of icVEP and GCIPL $(P>0.05)$. With the qualitative comparison, the sensitivity of icVEP was $80 \%$, and its specificity was $90 \%$. The sensitivity of GCIPL analysis was $72 \%$, and its specificity was $85 \%$. There was no significant difference between the sensitivitiesor specificities of icVEP and GCIPL analysis ( $P>0.05)$. Moreover, 30 (66.67\%) eyeshad similar resultsbetween icVEP and GCIPL analysis, and 15 (33.33\%) eyes had different results (7 eyes had abnormal results with GCIPL analysisbut normal results with icVEP, and8 eyes had normal results with GCIPL analysisbut abnormal results with icVEP).
\end{abstract}

Conclusions: The diagnostic power of icVEP was close to that of GCIPL analysis whether the comparison was based on the qualitative or quantitative data.

Keywords: Isolated-check visual evoked potential, Optical coherence tomography, Signal-to-noise ratios, Glaucomatous optic neuropathy

\section{Background}

Primary open angle glaucoma is a progressive optic neuropathy that is usually caused by high pressure inside the eye and characterized by gradual degeneration of the retinal ganglion cells (RGC) [1]. The most widely used method of functional assessment for diagnosing glaucoma is visual field analysis, which relies on behavioral

\footnotetext{
* Correspondence: zhaoyixi@163.com

Department of Out Patient Service, The Eye Hospital of Wenzhou Medical

University, Postal code: 325000, Wenzhou, Zhejiang Province, China
}

responses to detect functional deficits [2]. However, by the time these visual field deficits are detected, there is a significant reduction in the RGC population [3]. Therefore, examination by visual field analysis is not adequate for the early diagnosis of glaucoma.

Two advanced techniques have been introduced to the field to assess the RGC abnormalities associated with glaucoma. One is optical coherence tomography (OCT), which facilitates the quantitative and qualitative analyses of the structures of the retinal ganglion cell-inner 
plexiform layer (GCIPL) $[4,5]$. Another is isolated-check visual evoked potential (icVEP), which is designed to detect the transmission function of the magnocellular pathway, a neural pathway that is mainly managed by the RGCs [6]. The GCIPL analysis is used to detect early glaucoma by assessing the structural changes of macular RGCs [7-9] whereas the icVEP is used to diagnose early glaucoma by identifying the functional abnormalities of macular RGCs. Although GCIPL analysis and icVEP techniques target the same neuroretinal cells, the detection mechanisms of these two techniques are entirely different. This study investigated the above tests to determine which one performs better in the diagnosis of early glaucoma.

Therefore, the primary purpose of the current study is to compare the diagnostic power of icVEP and GCIPL analysis quantitatively and qualitatively at the RGC level in eyes with early primary open angle glaucoma.

\section{Methods \\ Patients}

Participants were recruited from the Eye Hospital of Wenzhou Medical University. All patients signed informed consent forms prior to participation. The Tenets of the Declaration of Helsinki were followed. This study was approved by the Ethics Committee of the Eye Hospital of Wenzhou Medical University. All participants underwent the necessary ophthalmic examinations, including visual acuity, slit-lamp biomicroscopy, refraction, gonioscopy, Goldmann applanation tonometry, visual field analysis, icVEP, and OCT. The visual field analysis was performed using the Humphrey Field Analyzer (HFA) II (model 750; Carl Zeiss Meditec, Inc.). To reduce learning effects, all participants took at least two HFA tests. The results of visual field analysis were considered reliable when fixation losses were $<20 \%$ and false positive and negative errors were $<33 \%$ [10].

The eyes were classified into the early glaucoma group or the control group. The inclusion criteria for the early glaucoma group were as follows: eyes with open anterior chamber angle and glaucomatous optic neuropathy (cup-disc ratio $\geq 0.6$ or vertical cup/disc diameter ratio asymmetry $\geq 0.2$ ), central corneal thickness (CCT) adjusted for untreated intraocular pressure $>21 \mathrm{mmHg}$, visual field defects on standard automated perimetry (SAP) (glaucoma hemifield test results that were outside normal limits; pattern standard deviation (PSD) with $p$ value $<5 \%$; or cluster of three or more non-edge points on the pattern deviation plot in a single hemi-field with $p$ values $<5 \%$, one of which must have a $p$ value $<1 \%$ [10]), and mean deviation of the visual field at $\leq 6 \mathrm{~dB}$. The inclusion criteria for the control group were as follows: no family history of glaucoma; intraocular pressure (IOP) <21 mmHg; a normal visual field; a central thickness of cornea $>500 \mathrm{~mm}$; normal optic nerve head; normal retinal nerve fibre layer.

Patients were excluded from this study if they met one of the following conditions: best corrected visual acuity $<20 / 30$; spectacle refraction $> \pm 5.00 \mathrm{D}$ sphere or $> \pm 2.00 \mathrm{D}$ cylinder; pupil diameter $<2.5 \mathrm{~mm}$; diseases that could lead to visual field loss or affect macular thickness; former ocular surgery (except for uncomplicated cataract surgery); or neurological diseases that may influence the icVEP results (e.g., amblyopia, ischemic optic neuropathy, etc.).

\section{Isolated-check visual evoked potential}

The icVEP was performed using the Neucodia visual electrophysiological diagnostic system (MKWH AMD, Huzhou Medconova Medical Technology, Inc.). At the beginning of icVEP testing, electrodes were connected to the scalp of patients using an electrolytic paste. Patients were instructed to listen for an auditory cue and to stare at a cross in the center of a computer screen, which was going to display a specific visual pattern. Elicited by this special picture, the subjects' cortical response was recorded by the Neucodia system, which presented the outcome as an electroencephalogram (EEG). This process totally took $2 \mathrm{~s}$ to finish (1 $\mathrm{s}$ each for EEG testing and recording). Next, the EEG was transformed into the fundamental frequency component (FFC), which is an important intermediate parameter in the examination. After eight separate runs, the instrument calculated the mean FFC and the radius of the 95\% confidence circle [6].

The signal-to-noise ratio (SNR) was defined as the ratio of the mean amplitude of FFC to the radius of the 95\% confidence circle. The SNR was the final number that was used to determine the presence of glaucomatous damage. SNR $\leq 1.0$ was defined as abnormal, whereas SNR $>1.0$ was defined as normal $[6,11]$ (Fig. 1). In this study, $15 \%$ positive-contrast (bright) condition pattern was used to differentiate between healthy participants and glaucoma patients.

To obtain reliable SNR, three methods were used in this test: automated noise detection, automated outlier analysis, and operator verification. The techniques for noise detection and outlier analysis were incorporated in the program of Neucodia system. If noise was detected by the device during the testing process, the elicited EEG was discarded and the program would prompt the operator to repeat the run. If noise was not detected, the EEG would be displayed on the screen and either be accepted or rejected by the operator depending on whether proper fixation was maintained during the test. After the eight FFCs were completed, outlier analysis was performed to identify whether one of the FFCs was an outlier relative to the other seven runs based on a statistical criterion. If a run was identified as an outlier, the device will discard it and prompt the operator to 


\section{A Control Age: 49 years}

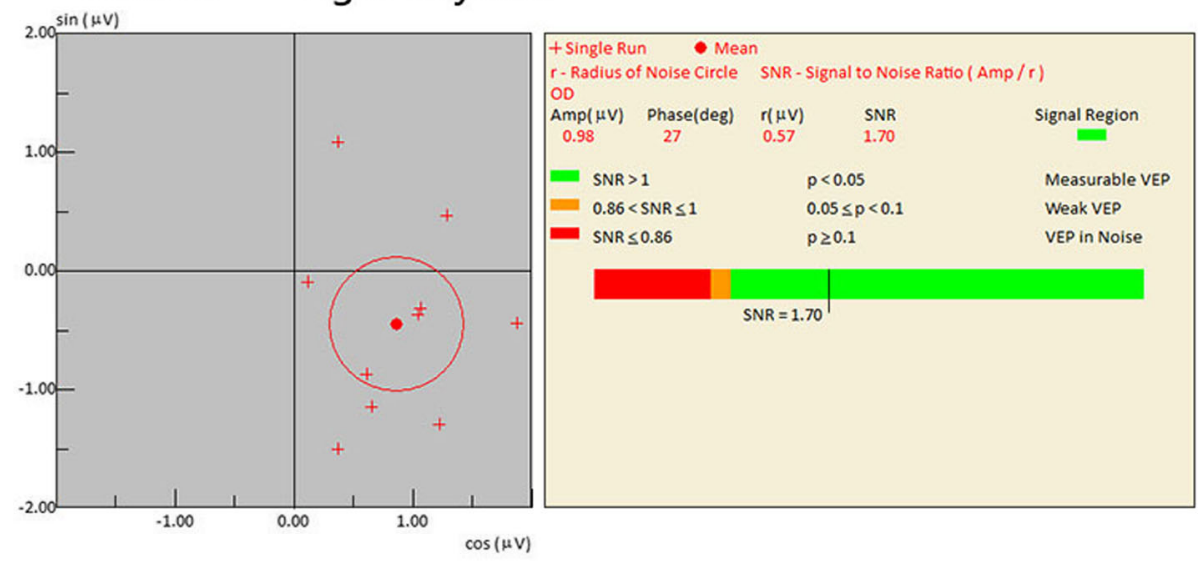

\section{B Patient Age: 74 years}
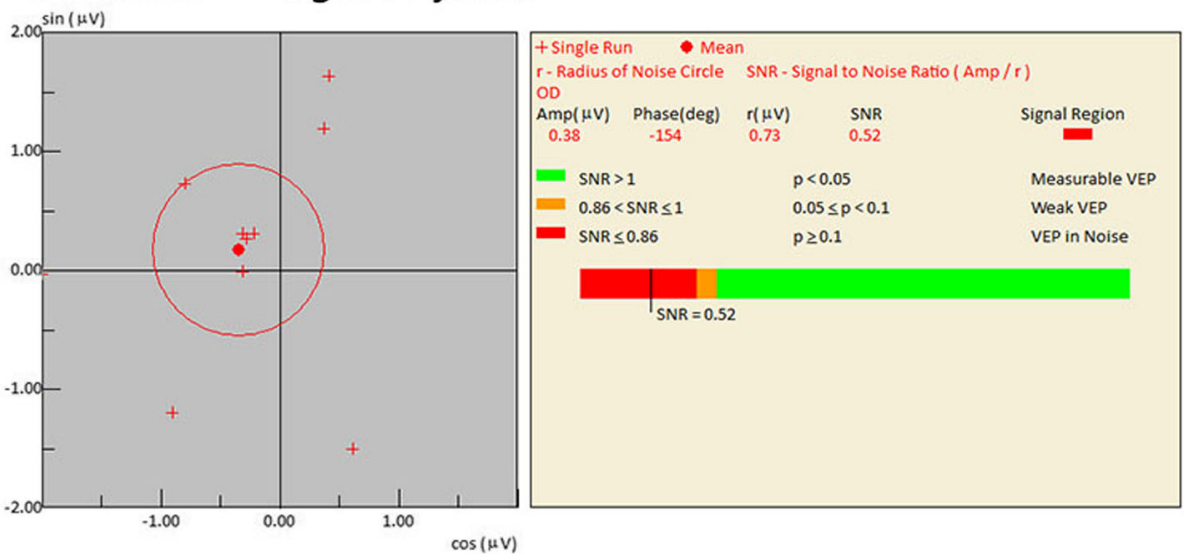

Fig. 1 Eight separate fundamental frequency components of the icVEP under the 15\% bright-check condition. a normal; b abnormal

repeat the test until eight qualified outcomes were collected. The process is shown in Fig. 2.

\section{Optical coherence tomography}

OCT examination was performed using the Cirrus HD-OCT (Carl Zeiss Meditec, Dublin, California) on the same day as icVEP testing. The operators who performed the OCT examination were different from those who performed the icVEP testing. Three OCT volume scans centered on the macula were performed for GCIPL analysis. The signal strength of the OCT was analyzed. Out of the three scans, only the one with the best signal strength was selected for GCIPL analysis.

The minimal (lowest GCIPL thickness), average, and sectoral (inferotemporal, superior, inferior, inferonasal, superonasal, superotemporal) thicknesses of the GCIPL were measured in an elliptical annulus around the fovea. Some studies have reported that the minimal GCIPL thickness assessment was better than the other two GCIPL analyses for diagnosing glaucoma $[4,5]$. Thus, the minimal GCIPL analysis was selected as the only indicator to assess the structural abnormalities in this study.

Furthermore, Cirrus software automatically organized the GCIPL values into three categories: within normal limits (green), borderline (yellow), and outside of normal limits (red) (Fig. 3). To maintain higher specificity, the eyes classified as "borderline (yellow)" were included in the category of "within normal limits" in this study $[12,13]$.

\section{Statistical analysis}

Continuous data were presented as mean \pm standard deviation (SD). The receiver operating characteristic (ROC) curves were used to determine the discriminatory capabilities of the tests between glaucomatous and healthy eyes. The area under the ROC curves (AUC) were evaluated for minimal GCIPL thickness and SNR values. These quantitative values of the AUCs were compared using the Clarke-Pearson method for paired data. 


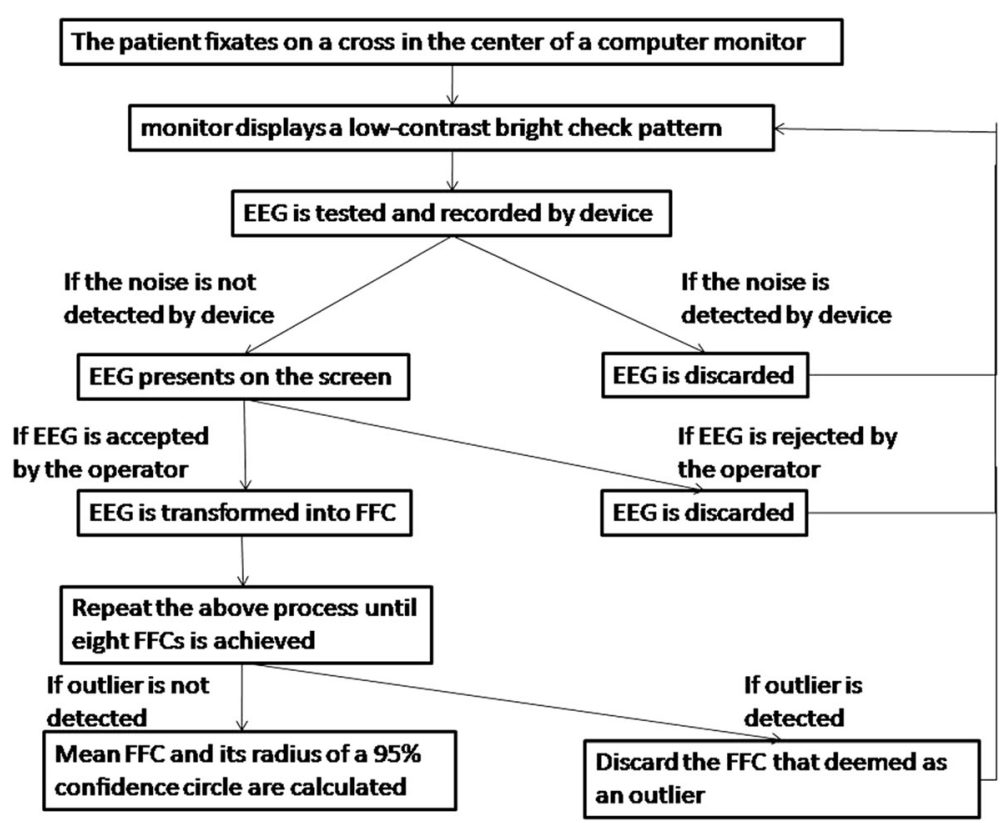

Fig. 2 The procedure for the icVEP test

The sensitivity was defined as the percentage of glaucomatous eyes that were abnormal in the structural or functional test. The specificity was defined as the percentage of control eyes that were normal in the structural or functional test. The comparison between sensitivity (or specificity) of icVEP and that of OCT was performed using the McNemar test. The level of significance was $p<0.05$.

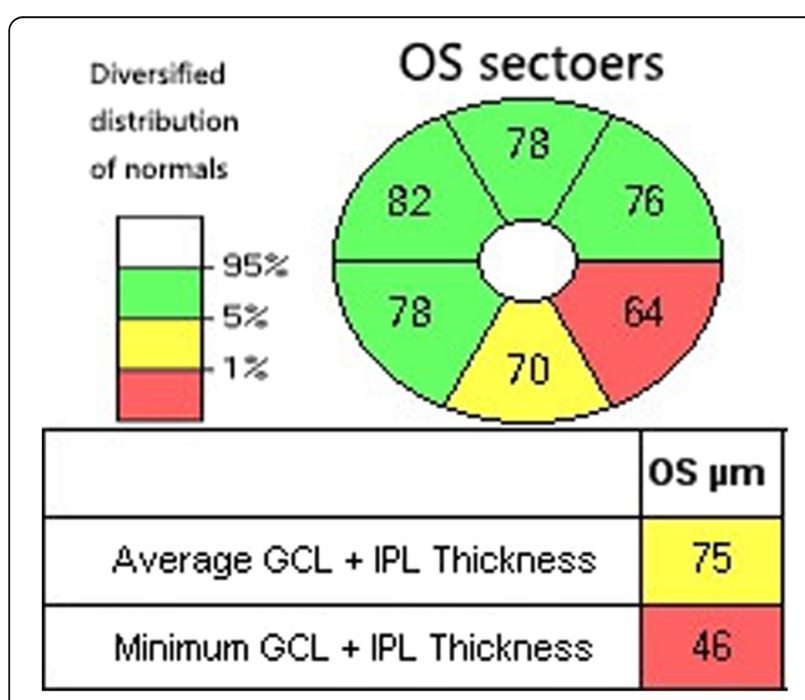

Fig. 3 Optical coherence tomography images of the left eye of a patient with early glaucoma. In this case, the GCIPL sectors in the temporal inferior area was outside of normal limits (red) and the inferior area was borderline (yellow). The minimum GCIPL was outside of normal limits (red), and the average GCIPL was borderline (yellow)

\section{Results}

Demographic characteristics of the study participants

A total of 45 eyes from 45 patients were enrolled in this study: 25 eyes were assigned to the early glaucoma group and 20 eyes were assigned to the control group. The range of ages was from 44 to 77 years $(60.96 \pm 9.56$ years). The demographic characteristics of the study participants are summarized in Table 1.

\section{Quantitative comparisons between icVEP and OCT}

Fig. 4 shows the ROC curves of icVEP and minimum GCIPL. Table 2 shows the AUCs, standard error, 95\% confidence interval, and significance of these two techniques. The AUC of icVEP (AUC $=0.892$ ) was higher than that of minimum GCIPL (AUC $=0.814$ ); however, the difference was not statistically significant $(\mathrm{z}=0.93$, $p=0.356)$.

\section{Qualitative comparisons between icVEP and OCT}

The classification of patients according to test results is listed in Table 3 . In the early glaucoma group, 20 eyes on icVEP and 18 eyes on OCT were abnormal. In the control group, 18 eyes on icVEP and 17 eyes on OCT were normal. Thus, the sensitivity of icVEP was $80 \%$, and its specificity was $90 \%$. The sensitivity of OCT was $72 \%$, and its specificity was $85 \%$. The McNemar test revealed that there was no statistically significant difference between the sensitivity (or specificity) of icVEP and minimum GCIPL (sensitivity, $p=0.75$; specificity, $p=1.00)$. In total, $30(66.67 \%)$ eyes had similar results between icVEP and GCIPL analysis, and 15 (33.33\%) 
Table 1 Demographic characteristics of the study participants

\begin{tabular}{lllll}
\hline Variable & Glaucoma group & Control group & Total & $P$-value \\
\hline Number of cases & 25 & 20 & 45 & $60.96 \pm 9.56$ \\
Age in years (mean \pm SD) & $59.24 \pm 9.88$ & $61.55 \pm 10.05$ & $29 / 16$ & 0.944 \\
Sex (male/female) & $17 / 8$ & $12 / 8$ & $-0.26 \pm 2.79$ & $-0.09 \pm 3.01$ \\
Spherical equivalents in diopters (mean \pm SD) & $0.4 \pm 3.22$ & 0.26 \\
\hline
\end{tabular}

eyes had different results (7 eyes had abnormal results with GCIPL analysis but normal results with icVEP, and 8 eyes had normal results with GCIPL analysis but abnormal results with icVEP).

\section{Discussion}

In this study, the quantitative comparison between the diagnostic performance of icVEP and that of OCT was performed using the ROC curve analyses. We discovered that although the absolute AUC value of SNR (derived from icVEP) was relatively higher than that of minimum GCIPL (derived from OCT) in this study, the statistical difference between these two AUC values was not significant. This result suggested that the diagnostic power of icVEP was similar to that of minimum GCIPL in the detection of early glaucoma when the comparison was based on quantitative data. However, the relatively higher AUC value of icVEP in this paper may not be purely by chance.

Several studies have supported that the AUC value of icVEP would be higher than that of OCT in the diagnosis of early glaucoma. First, studies have found that the individual GCIPL thickness varies with several factors, such as individual variation, age, and race [14]. This variation in GCIPL thickness presented a bias that would lower the diagnostic power of this technique in the diagnosis of glaucoma. Second, it was discovered that the RGCs with large diameter axons were preferentially damaged in early glaucoma $[15,16]$, and icVEP specialized in detecting the transmission function of the magnocellular pathway, which is mainly managed by the RGCs with large-diameter axons [17, 18]. However, the GCIPL analysis in OCT measured the retinal thicknesses from the ganglion cell layer to the inner plexiform layer [14], regardless of whether the RGCs with large diameter axons were included or not. This may also lower the diagnostic performance of GCIPL analysis in the detection of early glaucoma. Finally, it must be considered that in patients with glaucoma, there are two sources of functional impairment: one at the retinal level and another at the post-retinal level (such as at the lateral geniculate nucleus level [9]). icVEP examined the functional integrity of central vision at all levels of the visual pathway including the retina, optic nerve, optic radiations, and occipital cortex. However, OCT only detected the morphological abnormalities at the retinal level. These indicate that icVEP was more likely to identify glaucoma damage than OCT in the whole visual pathway. To sum up, the above three points strongly supported that icVEP would perform better than the minimum GCIPL analysis in the detection of early glaucoma. However, there was no statistically significant difference between the AUC

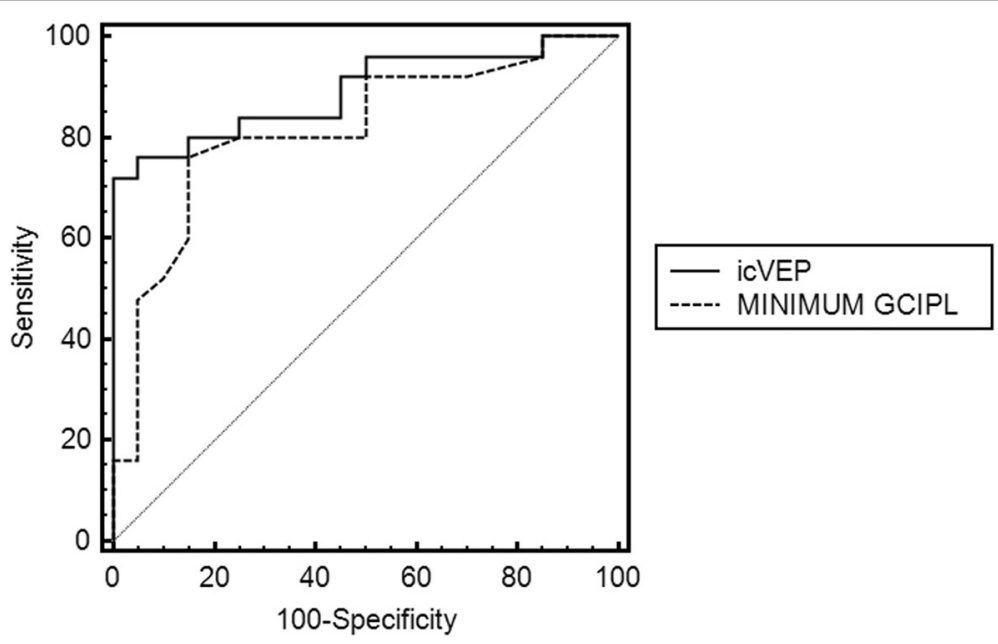

Fig. 4 The ROC curves of icVEP and minimum GCIPL 
Table 2 AUC, standard error, 95\% confidence interval and significance of icVEP and minimum GCIPL

\begin{tabular}{lllll}
\hline & AUC & Standard error & $95 \% \mathrm{Cl}$ & $P$ value \\
\hline icVEP & 0.892 & 0.0488 & 0.763 to 0.965 & $<0.0001$ \\
Minimum GCIPL & 0.814 & 0.0664 & 0.670 to 0.914 & $<0.0001$ \\
\hline
\end{tabular}

values of icVEP and minimum GCIPL in this study. Since sample size plays an important role in statistical analysis, further research is needed to determine whether the statistical result would change when a new study with a larger sample size is performed.

Qualitative comparisons between icVEP and minimum GCIPL were also performed in this study. In contrast to the icVEP test, the qualitative assessment of GCIPL analysis was not simply cutoff values of the macular thicknesses but were attributes given by the device, which took into account more relevant information (such as age and race) to assign a result to a given category. Therefore, the information obtained with the qualitative assessment of GICPL analysis was more robust than that of the quantitative assessment of GCIPL analysis. In this study, the sensitivity and specificity of these two examinations were calculated from the qualitative data. We discovered that there were no statistically significant differences between the sensitivities or specificities of these two techniques $(p>0.05)$. This revealed that the overall performance of icVEP was close to that of the minimum GCIPL when the comparison was based on the qualitative data. However, the disagreements between icVEP and minimum GCIPL were 15 (33.33\%) eyes, suggesting that icVEP detected some real abnormalities that minimum GCIPL did not and vice versa. This may be partially attributable to the variability of both techniques, but it is also possible that the functional abnormalities in early glaucoma did not manifest at the same time as the structural abnormalities. This viewpoint is supported by the findings of Higginbotham et al., who reported that structural progression accounted for nearly $60 \%$ of all conversions in early stage glaucoma, functional changes were responsible for $40 \%$, and both structural and functional abnormalities accounted for less than $15 \%$ [19]. Hence, the detection of structural or functional abnormalities alone would lead to missed diagnosis of early glaucoma. Therefore, a combined evaluation

Table 3 Contingency table of groupsversus test results

\begin{tabular}{llll}
\hline & & Early glaucoma group & Control group \\
\hline ICVEP & + & 20 & 2 \\
& - & 5 & 18 \\
Minimum GCIPL & + & 18 & 3 \\
& - & 7 & 17 \\
\hline${ }^{+}+{ }^{\prime \prime}$ = abnormality; "-" = normality
\end{tabular}

of structural and functional changes may improve the detection of early glaucoma.

The present study has several limitations. The patients assigned to the glaucoma group all had eyes with early stage glaucoma (the mean deviation of visual field was $\leq 6 \mathrm{~dB}$ ). Thus, the diagnostic performance of these two techniques would change if the patients with more serious stage glaucoma were enrolled in this study. Another limitation of this study was that the small sample size in the current study does not provide strong evidence for the results, which were based on the quantitative and qualitative comparisons between icVEP and OCT. Finally, peripheral visual function is known to be preferentially affected in the mild stage of glaucoma [20]. However, icVEP was designed to detect the central visual abnormalities of glaucoma. Further studies are needed to determine which examination is better in the diagnosis of early glaucoma: icVEP or techniques that were designed to identify the peripheral visual function, such as standard automated perimetry and multifocal visual evoked potential.

\section{Conclusions}

In conclusion, the diagnostic power of icVEP was close to that of GCIPL analysis whether the comparison was based on the qualitative or quantitative data in this study. Several studies have revealed that a combination of structural and functional assessments may improve the detection of early glaucoma [21]. Thus, both OCT and icVEP should be used in the future to search for an optimal tool in the detection of early glaucoma.

\section{Abbreviations}

AUC: Areas under the curve; CCT: Central corneal thickness;

EEG: Electroencephalogram; FFC: Fundamental frequency component;

GCILP: Retinal ganglion cell-inner plexiform layer; icVEP: Isolated-check visual evoked potential; IOP: Intraocular pressure; OCT: Optical coherence tomography; RGC: Retinal ganglion cells; ROC: Receiver operating characteristic; SAP: Standard automated perimetry; SD: Standard deviation; SNR: Signal-to-noise ratio

\section{Acknowledgement}

None.

\section{Funding}

This work was supported by the Plan of Wenzhou Science and Technology (No.Y20160439).

\section{Availability of data and materials}

The datasets generated and analysed during the current study are available from the corresponding author on reasonable request.

\section{Authors' contributions}

YXZ suggested concept of study and performed to conduct study. Collection, analysis and interpretation of data were performed by XWC. XWC wrote the manuscript. Both authors read and approved the final manuscript.

Competing interests

The authors do not have any conflict of interest to report and have no proprietary interest in any of the materials mentioned in this article. 


\section{Consent for publication}

Not applicable.

\section{Ethics approval and consent to participate}

This study was approved by the Ethics Committee of the Eye Hospital of Wenzhou Medical University and adhered to the tenets of the Declaration of Helsinki. All patients signed informed consent forms prior to participation.

\section{Publisher's Note}

Springer Nature remains neutral with regard to jurisdictional claims in published maps and institutional affiliations.

Received: 6 January 2017 Accepted: 15 May 2017

Published online: 22 May 2017

\section{References}

1. Cherecheanu AP, lancu R, Dascalu AM, Serban D, Pirvulescu R. Assessment of clinical and imagistic structural progression in glaucoma. Rom J Ophthalmol. 2015;59(3):129-32.

2. Nouri-Mahdavi K, Nassiri N, Giangiacomo A, Caprioli J. Detection of visual field progression in glaucoma with standard achromatic perimetry: a review and practical implications. Graefes Arch Clin Exp Ophthalmol. 2011:249(11):1593-616.

3. Moschos MM, Georgopoulos G, Chatziralli IP, Koutsandrea C. Multifocal VEP and OCT findings in patients with primary open angle glaucoma: a cross-sectional study. BMC Ophthalmol. 2012;12:34.

4. Larrosa JM, Moreno-Montañés J, Martinez-de-la-Casa JM, et al. A diagnostic calculator for detecting glaucoma on the basis of retinal nerve fiber layer, optic disc, and retinal ganglion cell analysis by optical coherence tomography. Invest Ophthalmol Vis Sci. 2015;56(11):6788-95.

5. Grecescu M. Optical coherence tomography versus visual evoked potentials in detecting subclinical visual impairment in multiple sclerosis. J Med Life. 2014;7(4):538-41.

6. Zemon V, Tsai JC, Forbes $\mathrm{M}$, et al. Novel electrophysiological instrument for rapid and objective assessment of magnocellular deficits associated with glaucoma. Doc Ophthalmol. 2008;117(3):233-43.

7. Rolle T, Dallorto L, Bonetti B. Retinal and macular ganglion cell count estimated with optical coherence tomography RTVUE-100 as a candidate biomarker for glaucoma. Invest Ophthalmol Vis Sci. 2016;57(13):5772-9.

8. Hirooka K, Izumibata S, Ukegawa K, Nitta E, Tsujikawa A. Estimating the rate of retinal ganglion cell loss to detect glaucoma progression: an observational cohort study. Medicine (Baltimore). 2016;95(30):e4209.

9. Raza AS, Hood DC. Evaluation of the structure-function relationship in glaucoma using a novel method for estimating the number of retinal ganglion cells in the human retina. Invest Ophthalmol Vis Sci. 2015;56(9):5548-56.

10. Rolle T, Manerba L, Lanzafame P, Grignolo FM. Diagnostic power of macular retinal thickness analysis and structure-function relationship in glaucoma diagnosis using SPECTRALIS OCT. Curr Eye Res. 2016;41(5):667-75.

11. Salim S, Childers K, Lupinacci AP, Hu GZ, Zemon V, Netland PA. Influence of pupil size and other test variables on visual function assessment using visual evoked potentials in normal subjects. Doc Ophthalmol. 2010;121(1):1-7.

12. Fortune B, Demirel S, Zhang $X$, et al. Comparing multifocal VEP and standard automated perimetry in high-risk ocular hypertension and early glaucoma. Invest Ophthalmol Vis Sci. 2007:48(3):1173-80.

13. Yum HR, Park SH, Park HY, Shin SY. Macular ganglion cell analysis determined by Cirrus HD optical coherence tomography for early detecting Chiasmal compression. PLoS One. 2016;11(4):e0153064.

14. Jia $Y$, Wei $E$, Wang $X$, et al. Optical coherence tomography angiography of optic disc perfusion in glaucoma. Ophthalmology. 2014;121(7):1322-32.

15. Quigley HAGR, Dunkelberger GRGWR. Chronic human glaucoma causing selectively greater loss of large optic nerve fibers. Ophthalmology. 1988;95:357-63.

16. Kerrigan-Baumrind LA QHA, Pease MEKDF, Mitchell RS. Number of ganglion cells in glaucoma eyes compared with threshold visual field tests in the same persons. Invest Ophthalmol Vis Sci. 2000:41:741-8.

17. Zemon VGJ, Welch J. Asymmetries in ON and OFF visual pathways of humans revealed using contrast-evoked cortical potentials. Vis Neurosci. 1988;1:145-50.

18. Zemon VGJ. Luminance contrast mechanisms in humans: visual evoked potentials and a nonlinear model. Vis Res. 2006;46:4163-80.
19. Higginbotham EJ, Gordon MO, Beiser JA, et al. The ocular hypertension treatment study: topical medication delays or prevents primary open-angle glaucoma in African American individuals. Arch Ophthalmol. 2004;122(6):813-20.

20. Levkovitch-Verbin H. Retinal ganglion cell apoptotic pathway in glaucoma: initiating and downstream mechanisms. Prog Brain Res. 2015;220:37-57.

21. Harasymowycz P, Kamdeu FA, Papamatheakis D. Screening for primary open-angle glaucoma in the developed world: are we there yet. Can J Ophthalmol. 2005:40(4):477-86.

\section{Submit your next manuscript to BioMed Central and we will help you at every step:}

- We accept pre-submission inquiries

- Our selector tool helps you to find the most relevant journal

- We provide round the clock customer support

- Convenient online submission

- Thorough peer review

- Inclusion in PubMed and all major indexing services

- Maximum visibility for your research

Submit your manuscript at www.biomedcentral.com/submit
) Biomed Central 